\title{
Optical-Model Potential and RPA in the Continuum
}

\author{
J. CUGNON* \\ Theoretical Nuclear Physics, University of Liège, \\ Sart Tilman, 4000 Liège 1, Belgium
}

Received April 22, 1971

The optical-model potential for nucleon-nucleus scattering is constructed theoretically in the framework of the RPA. Inelastic channels and resonant states contributions to the imaginary part differ from the TDA case. The origin of this difference is qualitatively discussed. A small background term is added to the real part.

\section{Introduction}

The shell model extended to the continuum provides in a useful tool to investigate the effect of the compound nucleus and the direct interactions on the optical-model potential ${ }^{1,2}$. Unfortunately, this shell-model theory is essentially a Tamm-Dancoff approximation (TDA) and neglects correlations in the target, at least for most numerical applications, where only $1 p-1 h$ excitations are taken into account ${ }^{3}$. To avoid these drawbacks and to describe collective excitations, a more elaborated model has been built up, which is the random phase approximation (RPA) extended to the continuum ${ }^{4-9}$. The purpose of this paper is to use this formalism to study the effect of the correlations on the optical-model potential. It is found that the resonant states and inelastic channels contributions to the imaginary part are influenced by the correlations in the ground state. Moreover a slowly varying (and probably small) term is added to the real part, due to the boson-boson interaction.

* Chercheur I.I.S.N.

1 Mahaux, C., Weidenmüller, H. A.: Shell-model theory of nuclear reactions. Amsterdam: North-Holland Publishing Co. 1969.

2 Cugnon, J.: Z. Physik 233, 133 (1970).

3 Saruis, A. M., Marangoni, M.: Nucl. Phys. A 132, 433 (1969).-Marangoni, M., Saruis, A. M.: Nucl. Phys. A 132, 649 (1969).

4 Dietrich, K., Hara, K.: Nucl. Phys. A 111, 392 (1968).

5 Lemmer, R. H., Veneroni, M.: Phys. Rev. 170, 883 (1968).

6 Hahne, F. J. W., Dover, C. B.: Nucl. Phys. A 135, 65 (1969).

7 Dietrich, K., Dover, C.: Z. Physik 221, 340 (1969).

8 Ginocchio, J. N., Schucan, T. H., Weidenmüller, H. A.: Phys. Rev. C 1, 55 (1970).

9 Dover, C. B., Hahne, F. J. W.: Nucl. Phys. A 150, 257 (1970). 
In Section 2 the RPA equations are recalled. Section 3 is devoted to the definition of the generalized optical-model potential and to its construction. In Section 4, the optical-model potential proper is derived, together with useful simple expressions obtained with the help of suitable statistical assumptions. The physical origin of the different terms of the opticalmodel potential is discussed. Finally, Section 6 contains our conclusions.

\section{The RPA Equations}

In this section, we recall briefly the equations of the RPA extended to the continuum and define our notation. We closely follow Ref. ${ }^{8}$.

\section{a) Definition of the Hamiltonian}

The RPA Hamiltonian is defined by:

$$
\mathscr{H}=E_{H F}+H_{0}+V
$$

where $E_{H F}$ is the expectation value of the total hamiltonian $H$ with respect to $H F$ ground state. The. $H F$ hamiltonian $h$ possesses bound states and scattering states whose wave functions are $w_{l j}^{n}\left(r, k_{n}\right), u_{l j}(r, k)$. The quantity $H_{0}$ is defined by:

with

$$
H_{0}=\sum_{s} E_{s} A_{s}^{+} A_{s}+\sum_{c} \int_{\varepsilon_{c}}^{\infty} d E E A_{c}^{+}(E) A_{c}(E)
$$

$$
A_{s}^{+}=a_{p}^{+} \bar{a}_{h}^{+}, \quad A_{c}^{+}(E)=a_{p}^{+}(E) \bar{a}_{h}^{+} .
$$

The operators $\bar{a}_{h}^{+}, a_{p}^{+}\left(\bar{a}_{h}, a_{p}\right)$ create (annihilate) a hole or a particle in the $H F$ (non correlated) ground state. When the particle is unbound, its energy $\varepsilon_{p}$ is related to the variable $E$ by $E=\varepsilon_{p}+\varepsilon_{c}$. The quantity $V$ is defined by:

$$
\begin{aligned}
V= & \sum_{s s^{\prime}} V_{s s^{\prime}}^{(1)} A_{s}^{+} A_{s^{\prime}}+\frac{1}{2}\left\{\sum_{s, c}^{\infty} \int_{\varepsilon_{c}}^{\infty} d E V_{s c}^{(1)}(E) A_{s}^{+} A_{c}(E)+\text { H. C. }\right\} \\
& +\sum_{c c^{\prime}} \int_{\varepsilon_{c}}^{\infty} d E \int_{\varepsilon_{c^{\prime}}}^{\infty} d E^{\prime} V_{c c^{\prime}}^{(1)}\left(E, E^{\prime}\right) A_{c}^{+}(E) A_{c}\left(E^{\prime}\right) \\
& +\frac{1}{2}\left\{\sum_{s s^{\prime}} V_{s s^{\prime}}^{(2)} A_{s}^{+} A_{s^{\prime}}^{+}+\text {H. C. }\right\} \\
& +\sum_{s c}\left\{\int_{\varepsilon_{c}}^{\infty} d E V_{s c}^{(2)}(E) A_{s}^{+} A_{c}^{+}(E)+\text { H. C. }\right\} \\
& +\frac{1}{2}\left\{\sum_{c c^{\prime}} \int_{\varepsilon_{c}}^{\infty} d E \int_{\varepsilon_{c^{\prime}}}^{\infty} d E^{\prime} V_{c c^{\prime}}^{(2)}\left(E, E^{\prime}\right) A_{c}^{+}(E) A_{c}^{+}\left(E^{\prime}\right)+\mathrm{H} . \mathrm{C} .\right\},
\end{aligned}
$$


where $V^{(1)}$ is the part of the two-body force which is responsible of $p-h$ scattering and where $V^{(2)}$ is the part which corresponds to the creation (or destruction) of two $p-h$ pairs.

Within the RPA, the operators $A_{s}^{+}, A_{s}, A_{c}^{+}(E), A_{c}(E)$ satisfy the usual boson commutation relations.

\section{b) Equations of Motion for the Scattering States}

Let $\Psi_{E}^{(+) c}$ a scattering state of the hamiltonian $\mathscr{H}$, with incoming waves in channel $c$ only, which is denoted by the upper index $(+) c$. This state can be related to the true ground state $\Psi_{0}$ of $\mathscr{H}$ by the equation

$$
\left|\Psi_{E}^{(+) c}\right\rangle=Q_{E}^{(+) c \dagger}\left|\Psi_{0}\right\rangle \text {. }
$$

Then, the operator $Q_{E}^{(+) \dagger}$ must obey the relation

$$
\left[\mathscr{H}, Q_{E}^{(+) c \dagger}\right]=E Q_{E}^{(+) c \dagger}, \quad\left[Q_{E}^{(+) c \dagger}, Q_{E^{\prime}}^{(+) c^{\prime}}\right]=\delta_{c c^{\prime}} \delta\left(E-E^{\prime}\right) .
$$

The quantity $Q_{E}^{(+) c \dagger}$ is written as linear combination of the creation and annihilation elementary boson operators:

$$
\begin{aligned}
Q_{E}^{(+) c^{\dagger}=}= & \sum_{s}\left[X_{E}^{c}(s) A_{s}^{+}-Y_{E}^{c}(s) A_{s}\right] \\
& +\sum_{c^{\prime}} \int_{\varepsilon_{c^{\prime}}}^{\infty} d E^{\prime}\left[X_{E}^{c}\left(c^{\prime}, E^{\prime}\right) A_{c^{\prime}}^{+}\left(E^{\prime}\right)-Y_{E}^{c}\left(c^{\prime}, E^{\prime}\right) A_{c^{\prime}}\left(E^{\prime}\right)\right] .
\end{aligned}
$$

The coefficients $X_{E}^{c}$ and $Y_{E}^{c}$ satisfy the following integral equations ${ }^{8}$ :

$$
\begin{aligned}
& \sum_{s^{\prime}}\left(\begin{array}{cc}
\left(E-E_{s}\right) \delta_{s s^{\prime}}-V_{s s^{\prime}}^{(1)} & -V_{s s^{\prime}}^{(2)} \\
V_{s s^{\prime}}^{(2)} & \left(E+E_{s}\right) \delta_{s s^{\prime}}+V_{s s^{\prime}}^{(1)}
\end{array}\right)\left(\begin{array}{l}
X_{E}^{c}\left(s^{\prime}\right) \\
Y_{E}^{c}\left(s^{\prime}\right)
\end{array}\right) \\
& =\sum_{c^{\prime} \varepsilon_{c^{\prime}}}^{\infty} d E^{\prime}\left(\begin{array}{cc}
V_{s c^{\prime}}^{(1)}\left(E^{\prime}\right) & V_{s c^{\prime}}^{(2)}\left(E^{\prime}\right) \\
-V_{s c^{\prime}}^{(2)}\left(E^{\prime}\right) & -V_{s c^{\prime}}^{(1)}\left(E^{\prime}\right)
\end{array}\right)\left(\begin{array}{c}
X_{E}^{c}\left(c^{\prime}, E^{\prime}\right) \\
Y_{E}^{c}\left(c^{\prime}, E^{\prime}\right)
\end{array}\right) \text {, } \\
& \left(\begin{array}{cc}
E-E^{\prime} & 0 \\
0 & E+E^{\prime}
\end{array}\right)\left(\begin{array}{l}
X_{E}^{c}\left(c^{\prime}, E^{\prime}\right) \\
Y_{E}^{c}\left(c^{\prime}, E^{\prime}\right)
\end{array}\right) \\
& -\sum_{s}\left(\begin{array}{cc}
V_{s c^{\prime}}^{(1)}\left(E^{\prime}\right) & V_{c^{\prime} s}^{(2)}\left(E^{\prime}\right) \\
-V_{c^{\prime} s}^{(2)}\left(E^{\prime}\right) & -V_{c^{\prime} s}^{(1)}\left(E^{\prime}\right)
\end{array}\right)\left(\begin{array}{c}
X_{E}^{c}(s) \\
Y_{E}^{c}(s)
\end{array}\right) \\
& -\sum_{c^{\prime \prime} e_{c^{\prime \prime}}}^{\infty} d E^{\prime \prime}\left(\begin{array}{cc}
V_{c^{\prime} c^{\prime \prime}}^{(1)}\left(E^{\prime}, E^{\prime \prime}\right) & V_{c^{\prime} c^{\prime \prime}}^{(2)}\left(E^{\prime}, E^{\prime \prime}\right) \\
-V_{c^{\prime} c^{\prime \prime}}^{(2)}\left(E^{\prime}, E^{\prime \prime}\right) & -V_{c^{\prime} c^{\prime \prime}}^{(1)}\left(E^{\prime}, E^{\prime \prime}\right)
\end{array}\right)\left(\begin{array}{c}
X_{E}^{c}\left(c^{\prime \prime}, E^{\prime \prime}\right) \\
Y_{E}^{c}\left(c^{\prime \prime}, E^{\prime \prime}\right)
\end{array}\right)=0 .
\end{aligned}
$$

They generalize the TDA equations which can be recovered by making $V^{(2)}=0$. Their solution gives a complete knowledge of the wave function. 


\section{The Generalized Optical Model}

We define the generalized optical-model wave function by:

$$
\rho_{E}^{c}(r)=\left\langle\varphi_{c}\left|\int_{\varepsilon_{c}}^{\infty} d E^{\prime} X_{E}^{c}\left(c, E^{\prime}\right) A_{c}^{+}\left(E^{\prime}\right)\right| \Psi_{0}\right\rangle .
$$

The integration indicated by the round bracket runs over all the coordinates except the radial one of the last particle. The function $\rho_{E}^{c}(r)$ has the same asymptotic behaviour as $\Psi_{E}^{(+) c}$ since this behaviour is completely contained in that part of $\Psi_{E}^{(+) c}$ which is projected in $\varphi_{c}$ in Eq. (3.1). Using the same arguments as in Ref. ${ }^{1}$, we then get:

$$
\rho_{E}^{c}(r)=\int_{\varepsilon_{c}}^{\infty} d E^{\prime} X_{E}^{c}\left(c, E^{\prime}\right) u_{c}\left(r, k_{c}^{\prime}\right) .
$$

( $u_{c} \equiv u_{l j}, l j$ are the quantum numbers associated with channel $c$ )

The Schrödinger equation corresponding to this wave function can be written as:

$$
(E-D) \rho_{E}^{c}(r)-\int_{0}^{\infty} d r^{\prime} \mathscr{V}_{E}^{c}\left(r, r^{\prime}\right) \rho_{E}^{c}\left(r^{\prime}\right)=0
$$

In this equation, $D$ denotes the radial component of the $H F$ hamiltonian $h=t+v_{0}$. The potential $v_{0}+\mathscr{V}_{E}^{c}\left(r, r^{\prime}\right)$ is the generalized optical-model potential. Eq. (3.3) can be rewritten in the shell-model representation by multiplying by $u_{c}\left(r, k^{\prime}\right)$ and integrating over $r$. One gets ${ }^{1}$ :

with

$$
\left(E-E^{\prime}\right) X_{E}^{c}\left(c, E^{\prime}\right)-\int_{\varepsilon_{c}}^{\infty} d E^{\prime \prime} \mathscr{V}_{E}^{c}\left(E^{\prime}, E^{\prime \prime}\right) X_{E}^{c}\left(c, E^{\prime \prime}\right)=0,
$$

Inversely, one has ${ }^{1}$

$$
\mathscr{V}_{E}^{c}\left(E^{\prime}, E^{\prime \prime}\right)=\int_{0}^{\infty} d r \int_{0}^{\infty} d r^{\prime} u_{c}\left(r, k_{c}^{\prime}\right) \mathscr{V}_{E}^{c}\left(r, r^{\prime}\right) u_{c}\left(r, k_{c}^{\prime \prime}\right) .
$$

$$
\mathscr{V}_{E}^{c}\left(r, r^{\prime}\right)=\int_{\varepsilon_{c}}^{\infty} d E^{\prime} \int_{\varepsilon_{c}}^{\infty} d E^{\prime \prime} u_{c}\left(r, k_{c}^{\prime}\right) \mathscr{V}_{E}^{c}\left(r, r^{\prime}\right) u_{c}\left(r, k_{c}^{\prime \prime}\right) .
$$

We construct now the generalized optical-model potential. For this we rewrite Eq. (2.8b) as:

$$
\begin{aligned}
\left(1-\mathrm{K}_{E}^{c}\right)\left(\begin{array}{c}
X_{E}^{c}\left(c^{\prime}, E^{\prime}\right) \\
Y_{E}^{c}\left(c^{\prime}, E^{\prime}\right)
\end{array}\right)= & \left(\begin{array}{c}
e^{i \delta_{c}} \delta_{c c^{\prime}} \delta\left(E-E^{\prime}\right) \\
0
\end{array}\right) \\
& +\mathrm{E}_{C}^{-1} \vee\left(\begin{array}{c}
X_{E}^{c}(s) \\
Y_{E}^{c}(s)
\end{array}\right)+\mathrm{E}_{C}^{-1} \vee\left(\begin{array}{c}
X_{E}^{c}\left(c^{\prime}, E^{\prime \prime}\right) \\
Y_{E}^{c}\left(c^{\prime}, E^{\prime \prime}\right)
\end{array}\right)
\end{aligned}
$$


where $K_{E}^{c}$ is a $2 \times 2$ matrix given explicitly by:

$$
\begin{aligned}
K_{E}^{c}\left(c^{\prime}, c^{\prime \prime}, E^{\prime}, E^{\prime \prime}\right)= & \left(\begin{array}{cc}
\frac{1}{E^{+}-E^{\prime}} & 0 \\
0 & \frac{1}{E+E^{\prime}}
\end{array}\right) \\
& \cdot\left(1-\delta_{c c^{\prime}} \delta_{c c^{\prime \prime}}\right)\left(\begin{array}{cc}
V_{c^{\prime} c^{\prime \prime}}^{(1)}\left(E^{\prime}, E^{\prime \prime}\right) & V_{c^{\prime} c^{\prime \prime}}^{(2)}\left(E^{\prime}, E^{\prime \prime}\right) \\
-V_{c^{\prime} c^{\prime \prime}}^{(2)}\left(E^{\prime}, E^{\prime \prime}\right) & -V_{c^{\prime} c^{\prime \prime}}^{(1)}\left(E^{\prime}, E^{\prime \prime}\right)
\end{array}\right),
\end{aligned}
$$

and where 1 is the $2 \times 2$ unit matrix. The matrix $V$ is the one written down in Eq. (3.7b) when it is comprised between quantities both containing a continuous variable $\left(E^{\prime}\right)$ like, for instance, in the third term of the r.h.s. of Eq. (3.7a). It has the form given in the r.h.s. of Eq. (2.8a) when comprised between two quantities, one containing a continuous variable $\left(E^{\prime}\right)$ and the other a discrete one $(s)$. This appears in the second term of the r.h.s. of Eq. (3.7a). The matrix $E_{C}^{-1}$ is given by:

$$
E_{C}^{-1}=\left(\begin{array}{cc}
\frac{1}{E^{+}-E^{\prime}} & 0 \\
0 & \frac{1}{E+E^{\prime}}
\end{array}\right) \text {. }
$$

In Eq. (3.7a) and in the following, summation over the variable $s$ or summation over $c^{\prime} \neq c$ and integration over $E^{\prime}$ are omitted. It is easy to see where they operate. In order to construct the generalized opticalmodel potential, we extract from Eq. (3.7a) the coefficients $X_{E}^{c}\left(c^{\prime}, E^{\prime}\right)$ and $Y_{E}^{c}\left(c^{\prime}, E^{\prime}\right)$ for $c^{\prime} \neq c$. We insert their value in Eq. (2.8a) and find an expression for the coefficients $X_{E}^{c}(c), Y_{E}^{c}(c)$ that we introduce in Eq. (2.8 b). Let $\mathrm{F}_{E}^{c}$ be the resolvent of $\mathrm{K}_{E}^{c}$ :

$$
\left(1+F_{E}^{c}\right)\left(1-K_{E}^{c}\right)=\left(1-K_{E}^{c}\right)\left(1+F_{E}^{c}\right)=1 \text {. }
$$

We readily get:

$$
\begin{aligned}
\left(\begin{array}{cc}
E-E^{\prime} & 0 \\
0 & E+E^{\prime}
\end{array}\right) & \left(\begin{array}{l}
X_{E}^{c}\left(c, E^{\prime}\right) \\
Y_{E}^{c}\left(c, E^{\prime}\right)
\end{array}\right) \\
& =\mathrm{V}^{e} \mathrm{D}^{c^{-1}}(E) \mathrm{V}^{e}\left(\begin{array}{l}
X_{E}^{c}\left(c, E^{\prime \prime}\right) \\
Y_{E}^{c}\left(c, E^{\prime \prime}\right)
\end{array}\right)+\mathrm{V}^{e}\left(\begin{array}{l}
X_{E}^{c}\left(c, E^{\prime \prime}\right) \\
Y_{E}^{c}\left(c, E^{\prime \prime}\right)
\end{array}\right),
\end{aligned}
$$

where effective interaction $V^{e}$ is given by

$$
\mathrm{V}^{e}=\mathrm{V}+\mathrm{V}\left(\mathbf{1}+\mathrm{F}_{E}^{c}\right) \mathrm{E}_{C}^{-1} \mathrm{~V}
$$

If $M$ denotes the number of boson states $A_{s}^{+}\left|\Psi_{0}\right\rangle$, the matrix $\mathrm{D}^{c}$ is a $2 M \times 2 M$ matrix acting between the latter states and given by:

$$
\mathrm{D}^{c}=\mathrm{E}_{S}-\mathrm{V}^{e}
$$


with

$$
\left(E_{S}\right)_{s s^{\prime}}=\left(\begin{array}{cc}
\left(E-E_{s}\right) \delta_{s s^{\prime}} & 0 \\
0 & \left(E+E_{s}\right) \delta_{s s^{\prime}}
\end{array}\right) .
$$

The last operation, absent in the $\mathrm{TDA}^{1,2}$, consists in eliminating the coefficient $Y_{E}^{c}\left(c, E^{\prime}\right)$ from Eq. (3.10).

We define $\mathrm{V}^{e X}$, $\mathrm{V}^{e Y}$ and $\mathrm{F}^{Y}$ by:

$$
\begin{gathered}
\mathrm{V}^{e X}=\mathrm{V}^{e}\left(\begin{array}{ll}
1 & 0 \\
0 & 0
\end{array}\right), \quad \mathrm{V}^{e Y}=\mathrm{V}^{e}\left(\begin{array}{ll}
0 & 0 \\
0 & 1
\end{array}\right), \\
\left(1-\mathrm{E}_{C}^{-1} \mathrm{~V}^{e X}\right)\left(1+\mathrm{F}^{\mathrm{Y}}\right)=\left(\mathbf{1}+\mathrm{F}^{\mathrm{Y}}\right)\left(1-\mathrm{E}_{c}^{-1} \mathrm{~V}^{e Y}\right)=\mathbf{1} .
\end{gathered}
$$

We write Eq. (3.10) in the form:

$$
\begin{aligned}
\left(\begin{array}{c}
X_{E}^{c}\left(c, E^{\prime}\right) \\
Y_{E}^{c}\left(c, E^{\prime}\right)
\end{array}\right)= & \left(\begin{array}{c}
e^{i \delta_{c}} \delta\left(E-E^{\prime}\right) \\
0
\end{array}\right)+\mathrm{E}_{C}^{-1} \mathrm{~V}^{e} \mathrm{D}^{c-1} \\
& \cdot\left[\mathrm{V}^{e X}\left(\begin{array}{c}
X_{E}^{c}\left(c, E^{\prime \prime}\right) \\
0
\end{array}\right)+\mathrm{V}^{e Y}\left(\begin{array}{c}
0 \\
Y_{E}^{c}\left(c, E^{\prime \prime}\right)
\end{array}\right)\right] \\
& +\mathrm{E}_{C}^{-1}\left[\mathrm{~V}^{e X}\left(\begin{array}{c}
X_{E}^{c}\left(c, E^{\prime \prime}\right) \\
0
\end{array}\right)+\mathrm{V}^{e Y}\left(\begin{array}{c}
0 \\
Y_{E}^{c}\left(c, E^{\prime \prime}\right)
\end{array}\right)\right]
\end{aligned}
$$

Applying on the left the operator $\left(1+\mathrm{F}^{Y}\right)$, we obtain with the help of Eq. $(3.13 b)$ :

$$
\begin{aligned}
& \left(\begin{array}{c}
X_{E}^{c}\left(c, E^{\prime}\right) \\
Y_{E}^{c}\left(c, E^{\prime}\right)
\end{array}\right) \\
& \quad=\left(1+\mathrm{F}^{Y}\right) \mathrm{E}_{C}^{-1} \mathrm{~V}^{e} \mathrm{D}^{c^{-1}}\left[\mathrm{~V}^{e X}\left(\begin{array}{c}
X_{E}^{c}\left(c, E^{\prime \prime}\right) \\
0
\end{array}\right)+\mathrm{V}^{e Y}\left(\begin{array}{c}
0 \\
Y_{E}^{c}\left(c, E^{\prime \prime}\right)
\end{array}\right)\right] \\
& \quad+\left(1+\mathrm{F}^{Y}\right) \mathrm{E}_{C}^{-1} \mathrm{~V}^{e X}\left(\begin{array}{c}
X_{E}^{c}\left(c, E^{\prime \prime}\right) \\
0
\end{array}\right) .
\end{aligned}
$$

Replacing this value in the last term of Eq. (3.14), we find

$$
\begin{aligned}
\left(\begin{array}{c}
X_{E}^{c}\left(c, E^{\prime}\right) \\
Y_{E}^{c}\left(c, E^{\prime}\right)
\end{array}\right)= & \left(\begin{array}{c}
e^{i \delta_{c}} \delta\left(E-E^{\prime}\right) \\
0
\end{array}\right) \\
& +\mathrm{E}_{C}^{-1}\left[\mathrm{~V}^{e} \mathrm{D}^{c^{-1}} \mathrm{~V}^{e X}+\mathrm{V}^{e X}+\mathrm{V}^{e Y}\left(1+\mathrm{F}^{Y}\right) \mathrm{E}_{C}^{-1} \mathrm{~V}^{e X}\right] \\
& \cdot\left(\begin{array}{c}
X_{E}^{c}\left(c, E^{\prime \prime}\right) \\
0
\end{array}\right)+\mathrm{E}_{C}^{-1}\left[1+\mathrm{V}^{e Y}\left(1+\mathrm{F}^{Y}\right) \mathrm{E}_{C}^{-1}\right] \\
& \cdot \mathrm{V}^{e} \mathrm{D}^{c^{-1}} \mathrm{~V}^{e Y}\left(\begin{array}{c}
0 \\
Y_{E}^{c}\left(c, E^{\prime \prime}\right)
\end{array}\right) .
\end{aligned}
$$


Multiplying by $V^{e Y}\left(E^{\prime}\right)$ and integrating over the variable $E^{\prime}$, we get:

$$
\begin{aligned}
\int_{e_{c}}^{\infty} d E^{\prime} & V^{e Y}\left(E^{\prime}\right)\left(\begin{array}{c}
0 \\
Y_{E}^{c}\left(c, E^{\prime}\right)
\end{array}\right) \\
= & \Delta^{c^{-1}} V^{e Y} \mathrm{E}_{\mathrm{C}}^{-1}\left\{\mathrm{~V}^{e X}+\mathrm{V}^{e} \mathrm{D}^{c^{-1}}\left[\mathbf{1}+\mathrm{V}^{e Y}\left(\mathbf{1}+\mathrm{F}^{Y}\right) \mathrm{E}_{C}^{-1}\right] \mathrm{V}^{e X}\right\} \\
& \cdot\left(\begin{array}{c}
X_{E}^{c}\left(c, E^{\prime \prime}\right) \\
0
\end{array}\right),
\end{aligned}
$$

with

$$
A^{c}=1-V^{e Y} E_{C}^{-1}\left[1+V^{e Y}\left(1+F^{Y}\right) E_{C}^{-1}\right] V^{e} D^{c^{-1}} .
$$

We insert the value (3.17a) in Eq. (3.16) and multiply this equation by $E_{C}$. Taking the first component, we obtain Eq. (3.4) with

$$
\begin{aligned}
\mathscr{V}_{E_{s}}^{c}\left(E_{\alpha^{\prime}}^{\prime} E^{\prime \prime}\right)= & (1,0)\left\{\mathrm{V}^{e}\left(E^{\prime}\right) \mathrm{D}^{c^{-1}}(E) \mathrm{V}^{e}\left(E^{\prime \prime}\right)+\mathrm{V}_{c c}^{e}\left(E^{\prime}, E^{\prime \prime}\right)\right. \\
& \left.+\mathrm{V}^{e}\left(E^{\prime}\right) \mathrm{D}^{c^{-1}}(E) \mathrm{V}^{e Y}\left(1+\mathrm{F}^{Y}\right) \mathrm{E}_{C}^{-1} \mathrm{~V}^{e}\left(E^{\prime \prime}\right)\right\}\left(\begin{array}{l}
1 \\
0
\end{array}\right) \\
& +(1,0)\left\{\left[1+\mathrm{V}^{e X}\left(1+\mathrm{F}^{Y}\right) \mathrm{E}_{C}^{-1}\right] \mathrm{V}^{e} \mathrm{D}^{c^{-1}}(E) A^{c^{-1}} \mathrm{~V}^{e Y} \mathrm{E}_{C}^{-1}\right\} \\
& \cdot\left\{\mathrm{V}^{e}\left(E^{\prime \prime}\right)+\mathrm{V}^{e} \mathrm{D}^{c^{-1}}(E)\left[1+\mathrm{V}^{e Y} \mathrm{D}^{c^{-1}}(E)\right.\right. \\
& \left.\left.\cdot\left(1+\mathrm{V}^{e Y}\left(1+\mathrm{F}^{Y}\right) \mathrm{E}_{c}^{-1}\right)\right] \mathrm{V}^{e}\left(E^{\prime \prime}\right)\right\}\left(\begin{array}{l}
1 \\
0
\end{array}\right)
\end{aligned}
$$

Let us define the operators $V^{Y}, W^{Y}$ and $\mathscr{D}^{c}$ by

$$
\begin{gathered}
\mathrm{V}^{Y}=\mathrm{V}^{e Y}\left(\mathbf{1}+\mathrm{F}^{Y}\right) \mathrm{E}_{\mathrm{C}}^{-1} \mathrm{~V}^{e}, \quad \mathrm{~W}^{Y}=\mathrm{V}^{e}+\mathrm{V}^{Y}, \\
\mathscr{D}^{c}=\mathrm{D}^{c}-\mathrm{V}^{e X} \mathrm{E}_{\mathrm{C}}^{-1} \mathrm{~W}^{Y} .
\end{gathered}
$$

We can write Eq. (3.18) in a more compact form:

$$
\begin{aligned}
\mathscr{V}_{E}^{c}\left(E^{\prime}, E^{\prime \prime}\right)= & (1,0)\left\{\mathrm{V}_{c c}^{e}\left(E^{\prime}, E^{\prime \prime}\right)+\mathrm{V}^{e}\left(E^{\prime}\right) \mathrm{D}^{c^{-1}}(E) \mathrm{V}^{e}\left(E^{\prime \prime}\right)\right\}\left(\begin{array}{l}
1 \\
0
\end{array}\right) \\
& +(1,0)\left\{\mathrm{V}^{e}\left(E^{\prime}\right) \mathrm{D}^{c^{-1}}(E) \mathrm{V}^{Y}\left(E^{\prime \prime}\right)+\left[\mathrm{V}^{e}\left(E^{\prime}\right)+\mathrm{V}^{Y}\left(E^{\prime}\right)\right]\right. \\
& +\mathscr{D}^{c^{-1}}(E) \mathrm{V}^{e Y} \mathrm{E}_{C}^{-1}\left[\mathrm{~V}^{e}\left(E^{\prime \prime}\right)+\mathrm{V}^{e} \mathrm{D}^{c^{-1}}(E) \mathrm{V}^{e}\left(E^{\prime \prime}\right)\right. \\
& \left.\left.+\mathrm{V}^{e} \mathrm{D}^{c^{-1}}(E) \mathrm{V}^{Y}\left(E^{\prime \prime}\right)\right]\right\}\left(\begin{array}{l}
1 \\
0
\end{array}\right) .
\end{aligned}
$$

The generalized optical-model potential has a fairly complicated form. This complication arises from two sources: i) the continuumcontinuum interaction (already present in the TDA) which implies the introduction of the effective interaction $V^{e}$. ii) the RPA itself, which leads to the following two features. Firstly, the interactions are described 
now by matrices. Secondly, the states $\left|Z_{E}^{c}\right\rangle=A_{E}^{c}\left|\Psi_{0}\right\rangle$ are virtually excited from the intermediate states $A_{\boldsymbol{E}}^{c^{\prime}+}\left|\Psi_{0}\right\rangle, c^{\prime} \neq c$, and $A_{s}^{+}\left|\Psi_{0}\right\rangle$ and they must decay back to those states, since they do not contribute to the asymptotic behaviour of $\left|\Psi_{E}^{c(+)}\right\rangle$. This effect is responsible for the presence of the second term in curly brackets in Eq. (3.19c) (see Eq. (3.19a)).

The operators $F^{c}$ and $F^{Y}$ can be expanded in Born series

$$
\begin{aligned}
& \mathrm{F}^{c}=\mathrm{K}^{c}+\mathrm{K}^{c^{2}}+\mathrm{K}^{c^{3}}+\cdots, \\
& \mathrm{F}^{\mathrm{Y}}=\mathrm{K}^{\mathrm{Y}}+\mathrm{K}^{\mathrm{Y}^{2}}+\mathrm{K}^{\mathrm{Y}^{3}}+\cdots
\end{aligned}
$$

These series converge if there exist no narrow single-particle resonances in the $H F$ potential well. The divergence of the Born series, if it occurs, can be removed by using a method similar to those described in Ref. ${ }^{8}$.

\section{The Optical-Model Potential}

In this section, we derive the optical-model potential and some useful expressions by extracting the driving terms.

It is well known that in the absence of the single-particle resonances ${ }^{1}$, the optical-model potential can be written in shell-model representation as:

with

$$
\mathscr{\mathscr { V }}_{E}^{\mathrm{opt}(c)}\left(E^{\prime}, E^{\prime \prime}\right)=v_{0}\left(E^{\prime}, E^{\prime \prime}\right)+\tilde{\mathscr{V}}_{E}^{c}\left(E^{\prime}, E^{\prime \prime}\right)
$$

$$
\tilde{\mathscr{V}}_{E}^{c}\left(E^{\prime}, E^{\prime \prime}\right)=\mathscr{\mathscr { V }}_{E+i I}^{c}\left(E^{\prime}, E^{\prime \prime}\right) .
$$

We write then explicitly

with

$$
\begin{aligned}
\mathscr{V}_{E}^{\mathrm{opt}(c)}\left(E^{\prime}, E^{\prime \prime}\right)= & v_{0}\left(E^{\prime}, E^{\prime \prime}\right)+V_{c c}^{e(11)}\left(E^{\prime}, E^{\prime \prime}\right) \\
& +\mathscr{V}_{E}^{c N}\left(E^{\prime}, E^{\prime \prime}\right)+\mathscr{V}_{E}^{(1)}\left(E^{\prime}, E^{\prime \prime}\right),
\end{aligned}
$$

$$
\begin{gathered}
V_{c c}^{e}\left(E^{\prime}, E^{\prime \prime}\right)=\left(\begin{array}{ll}
V_{c c}^{e(11)} & V_{c c}^{e(12)} \\
V_{c c}^{e(21)} & V_{c c}^{e(22)}
\end{array}\right), \\
\mathscr{V}_{E}^{c N}\left(E^{\prime}, E^{\prime \prime}\right)=\sum_{s s^{\prime}}\left(V_{c s}^{e(11)}\left(E^{\prime}\right), V_{c s}^{e(12)}\left(E^{\prime}\right)\right)\left(D^{c^{-1}}(E+i I)\right)_{s s^{\prime}} \\
\cdot\left(\begin{array}{l}
V_{s^{\prime} c}^{e(11)}\left(E^{\prime \prime}\right) \\
V_{s^{\prime} c}^{e(21)}\left(E^{\prime \prime}\right)
\end{array}\right)
\end{gathered}
$$




$$
\begin{aligned}
& \mathscr{V}_{E}^{(1)}\left(E^{\prime}, E^{\prime \prime}\right)= \sum_{s s^{\prime}}\left(V_{c s}^{e(11)}\left(E^{\prime}\right), V_{c s}^{e(12)}\left(E^{\prime}\right)\right) \\
& \cdot\left(D^{c^{-1}}(E+i I)\right)_{s s^{\prime}}\left(\begin{array}{l}
V_{s^{\prime} c}^{Y(11)}\left(E^{\prime \prime}\right) \\
V_{s^{\prime} c}^{Y(21)}\left(E^{\prime \prime}\right)
\end{array}\right) \\
&+\sum_{s s^{\prime}}\left(W_{c s}^{Y(11)}, W_{c s}^{Y(12)}\right)\left(\mathscr{D}^{c^{-1}}(E+i I)\right)_{s s^{\prime}}\left(\begin{array}{l}
F_{s^{\prime} c}^{(11)} \\
F_{s^{\prime} c}^{(21)}
\end{array}\right) \\
&+\sum_{\substack{s s^{\prime} \\
s^{\prime \prime} s^{\prime \prime \prime}}}\left(W_{c s}^{Y(11)}, W_{c s}^{Y(12)}\right)\left(\mathscr{D}^{c^{-1}}(E+i I)\right)_{s s^{\prime}} \\
& \cdot F_{s^{\prime} s^{\prime \prime}}\left(D^{c^{-1}}(E+i I)\right)_{s^{\prime \prime} s^{\prime \prime \prime}}\left(\begin{array}{l}
W_{s^{\prime \prime \prime} c}^{Y(11)} \\
W_{s^{\prime \prime \prime} c}^{Y(21)}
\end{array}\right) \\
& \mathrm{F}=\mathrm{V}^{e Y} E_{C}^{-1} V^{e} .
\end{aligned}
$$

The term $\mathscr{V}_{E}^{C N}\left(E^{\prime}, E^{\prime \prime}\right)$ contains most of the effect of the compound resonant states. For simplicity, let us make in it $V^{e}=V$. The matrix $D^{c}$ can be put the form:

with

$$
D^{c}=\left(\begin{array}{rr}
A & C \\
-\tilde{C} & B
\end{array}\right)
$$

$$
\begin{aligned}
& A_{s s^{\prime}}=\left(E-E_{s}\right) \delta_{s s^{\prime}}-V_{s s^{\prime}}^{(1)}-\sum_{c^{\prime} \neq c} \int_{\varepsilon_{c^{\prime}}}^{\infty} d E^{\prime} \frac{V_{s c^{\prime}}^{(1)}\left(E^{\prime}\right) V_{c^{\prime} s^{\prime}}^{(1)}\left(E^{\prime}\right)}{E^{+}-E^{\prime}} \\
& +\sum_{c^{\prime} \neq c} \int_{\varepsilon_{c^{\prime}}}^{\infty} d E^{\prime} \frac{V_{s c^{\prime}}^{(2)}\left(E^{\prime}\right) V_{c^{\prime} s^{\prime}}^{(2)}\left(E^{\prime}\right)}{E+E^{\prime}} \\
& B_{s s^{\prime}}=\left(E+E_{s}\right) \delta_{s s^{\prime}}+V_{s s^{\prime}}^{(1)}+\sum_{c^{\prime} \neq c} \int_{c c_{c^{\prime}}}^{\infty} \frac{V_{s c^{\prime}}^{(2)}\left(E^{\prime}\right) V_{c^{\prime} s^{\prime}}^{(2)}\left(E^{\prime}\right)}{E^{+}-E^{\prime}} d E^{\prime} \\
& -\sum_{c^{\prime} \neq c} \int_{\varepsilon_{\varepsilon^{\prime}}}^{\infty} d E^{\prime} \frac{V_{s c^{\prime}}^{(1)}\left(E^{\prime}\right) V_{c^{\prime} s^{\prime}}^{(1)}\left(E^{\prime}\right)}{E+E^{\prime}} \\
& C_{s s^{\prime}}=-V_{s s^{\prime}}^{(2)}-\sum_{c^{\prime} \neq c} \int_{c \varepsilon_{c^{\prime}}}^{\infty} \frac{V_{s c^{\prime}}^{(1)}\left(E^{\prime}\right) V_{c^{\prime} s^{\prime}}^{(2)}\left(E^{\prime}\right)}{E^{+}-E^{\prime}} d E^{\prime} \\
& +\sum_{c^{\prime} \neq c} \int_{c}^{\infty} \frac{V_{s c^{\prime}}^{(2)}\left(E^{\prime}\right) V_{s^{\prime} c^{\prime}}^{(1)}\left(E^{\prime}\right)}{E+E^{\prime}}
\end{aligned}
$$

The matrix $D^{c^{-1}}$ can be written in the form ${ }^{8}$

$$
\mathrm{D}^{c^{-1}}=\left(\begin{array}{ll}
\mathrm{A}^{\prime} & \mathrm{C}^{\prime} \\
\mathrm{D}^{\prime} & \mathrm{B}^{\prime}
\end{array}\right),
$$


with

$$
\begin{aligned}
& A^{\prime}=d^{-1}, \quad=A-C B^{-1} \tilde{C}, \\
& C^{\prime}=-{ }^{-1} C B^{-1}, \quad D^{\prime}=B^{-1} C d^{-1}, \\
& B^{\prime}=B^{-1}-B^{-1} \tilde{C} d^{-1} C B^{-1} .
\end{aligned}
$$

If we define the quantities $\gamma_{s c}^{E}\left(E^{\prime}\right)$ by:

$$
\gamma_{s c}^{E}\left(E^{\prime}\right)=V_{s c}^{(1)}\left(E^{\prime}\right)+\sum_{s^{\prime} s^{\prime \prime}}(C(E))_{s s^{\prime}}\left(B^{-1}(E)\right)_{s^{\prime} s^{\prime \prime}} V_{s^{\prime \prime} c}^{(2)}\left(E^{\prime}\right)
$$

the quantity $\mathscr{V}_{E}^{C N}\left(E^{\prime}, E^{\prime \prime}\right)$ is given by:

$$
\begin{aligned}
\mathscr{V}_{E}^{C N}\left(E^{\prime}, E^{\prime \prime}\right)= & \sum_{s s^{\prime}} \gamma_{c s}^{E}\left(E^{\prime}\right)\left(d^{-1}(E+i I)\right)_{s s^{\prime}} \gamma_{s^{\prime} c}^{E}\left(E^{\prime \prime}\right) \\
& -\sum_{s s^{\prime}} V_{c s}^{(2)}\left(E^{\prime}\right)\left(B^{-1}(E)\right)_{s s^{\prime}} V_{s^{\prime} c}^{(2)}\left(E^{\prime \prime}\right) .
\end{aligned}
$$

One can get a simplified expression for $\gamma_{s c}^{E}\left(E^{\prime}\right)$ by taking only second order terms in $V$ and writing

$$
\gamma_{s c}^{E}\left(E^{\prime}\right)=V_{s c}^{(1)}\left(E^{\prime}\right)-\sum_{s^{\prime}} \frac{V_{s s^{\prime}}^{(2)}}{E+E_{s^{\prime}}} V_{s^{\prime} c}^{(2)}\left(E^{\prime}\right)
$$

The continuum-continuum effect on the quantities $\gamma$ may be introduced by replacing in Eq. (4.7b) the quantity $V$ by $V^{e \star}$.

Since the non-diagonal terms in the matrix $d$ have statistically varying signs, one may assume they have mutually cancellating effects. One has roughly:

$$
\mathscr{V}_{E}^{C N} \approx \sum_{s} \frac{\gamma_{c s}\left(E^{\prime}\right) \gamma_{s c}\left(E^{\prime \prime}\right)}{E+i I-E_{s}}-\sum_{s} \frac{V_{c s}^{(2)}\left(E^{\prime}\right) V_{s c}^{(2)}\left(E^{\prime \prime}\right)}{E+E_{s}}
$$

If the mean separating distance $d$ between successive $E_{s}$ 's is less than the averaging $I$, Eq. (4.15) reduces to:

$$
\mathscr{V}_{E}^{C N} \approx-i \pi \frac{\overline{\gamma_{c s}\left(E^{\prime}\right) \gamma_{s c}\left(E^{\prime \prime}\right)^{s}}}{d}-\sum_{s} \frac{V_{c s}^{(2)}\left(E^{\prime}\right) V_{s c}^{(2)}\left(E^{\prime \prime}\right)}{E+E_{s}} .
$$

Let us now discuss the second term of the r.h.s. of Eq. (4.3). The quantity $V^{e}$ is the main term due to the continuum-continuum interactions. It can be expanded as follows:

$$
V^{e}=V+V E_{c}^{-1} V+V E_{c}^{-1} V E_{c}^{-1} V+\cdots
$$

* $V^{e}$ must have the same structure as $V$ since it is true at each order of the perturbative series for $v^{e}$. 
or more explicitly

$$
\begin{aligned}
V_{c c}^{e}= & \left(\begin{array}{cc}
V_{c c}^{(1)}\left(E^{\prime}, E^{\prime \prime}\right) & V_{c c}^{(2)}\left(E^{\prime}, E^{\prime \prime}\right) \\
-V_{c c}^{(2)}\left(E^{\prime}, E^{\prime \prime}\right) & -V_{c c}^{(1)}\left(E^{\prime}, E^{\prime \prime}\right)
\end{array}\right) \\
& +\sum_{c^{\prime} \neq c \varepsilon_{\varepsilon^{\prime}}} \int^{\infty} d E^{\prime \prime \prime}\left(\begin{array}{cc}
V_{c c^{\prime}}^{(1)}\left(E^{\prime}, E^{\prime \prime \prime}\right) & V_{c c^{\prime}}^{(2)}\left(E^{\prime}, E^{\prime \prime \prime}\right) \\
-V_{c c^{\prime}}^{(2)}\left(E^{\prime}, E^{\prime \prime \prime}\right) & -V_{c c^{\prime}}^{(1)}\left(E^{\prime}, E^{\prime \prime \prime}\right)
\end{array}\right) \\
& \cdot\left(\begin{array}{cc}
\frac{1}{E^{+}-E^{\prime \prime \prime}} & 0 \\
0 & \frac{1}{E+E^{\prime \prime \prime}}
\end{array}\right)\left(\begin{array}{cc}
V_{c^{\prime} c}^{(1)}\left(E^{\prime \prime \prime}, E^{\prime \prime}\right) & V_{c^{\prime} c}^{(2)}\left(E^{\prime \prime \prime}, E^{\prime \prime}\right) \\
-V_{c^{\prime} c}^{(2)}\left(E^{\prime \prime \prime}, E^{\prime \prime}\right) & -V_{c^{\prime} c}^{(1)}\left(E^{\prime \prime \prime}, E^{\prime \prime}\right)
\end{array}\right)+\cdots
\end{aligned}
$$

In second order in $V$, we get:

$$
\begin{aligned}
V_{c c}^{e(11)}= & V_{c c}^{(1)}\left(E^{\prime}, E^{\prime \prime}\right)+\sum_{c^{\prime} \neq c} \int_{\varepsilon_{c^{\prime}}}^{\infty} d E^{\prime \prime \prime} \frac{V_{c c^{\prime}}^{(1)}\left(E^{\prime}, E^{\prime \prime \prime}\right) V_{c^{\prime} c}^{(1)}\left(E^{\prime \prime \prime}, E^{\prime \prime}\right)}{E^{+}-E^{\prime \prime \prime}} \\
& -\sum_{c^{\prime} \neq c} \int_{\varepsilon_{c^{\prime}}}^{\infty} d E^{\prime \prime \prime} \frac{V_{c c^{\prime}}^{(2)}\left(E^{\prime}, E^{\prime \prime \prime}\right) V_{c^{\prime} c}^{(2)}\left(E^{\prime \prime \prime}, E^{\prime \prime}\right)}{E+E^{\prime \prime \prime}} .
\end{aligned}
$$

The third term in the r.h.s. is the proper contribution of the RPA to the pure direct processes (in second order in $V$ ), since the first two are present in TDA. By taking the non-hermitian contribution of the RPA to the third order term of expansion (4.10a) and a small part of the fourth order term, one may get an expression similar to Eq. (4.9a):

$$
\begin{aligned}
V_{c c}^{e(11)}= & V_{c c}^{(1)}\left(E^{\prime}, E^{\prime \prime}\right)+\sum_{c^{\prime} \neq c} \int_{c \varepsilon_{c^{\prime}}}^{\infty} d E^{\prime \prime \prime} \frac{\zeta_{c c^{\prime}}^{(1)}\left(E^{\prime}, E^{\prime \prime \prime}\right) \zeta_{c^{\prime} c}^{(1)}\left(E^{\prime \prime \prime}, E^{\prime \prime}\right)}{E^{+}-E^{\prime \prime \prime}} \\
& -\sum_{c^{\prime} \neq c} \int_{\varepsilon_{c^{\prime}}}^{\infty} d E^{\prime \prime \prime} \frac{V_{c c^{\prime}}^{(2)}\left(E^{\prime}, E^{\prime \prime \prime}\right) V_{c^{\prime} c}^{(2)}\left(E^{\prime \prime \prime}, E^{\prime \prime}\right)}{E+E^{\prime \prime \prime}},
\end{aligned}
$$

with

$$
\begin{aligned}
\zeta_{c c^{\prime}}^{(1)}\left(E^{\prime}, E^{\prime \prime}\right)= & V_{c c^{\prime}}^{(1)}\left(E^{\prime}, E^{\prime \prime}\right) \\
& -\sum_{c^{\prime \prime} \neq c \varepsilon_{\varepsilon_{c}^{\prime \prime}}}^{\infty} d E^{\prime \prime \prime} \frac{V_{c c^{\prime \prime}}^{(2)}\left(E^{\prime}, E^{\prime \prime \prime}\right) V_{c^{\prime \prime} c^{\prime}}^{(2)}\left(E^{\prime \prime \prime}, E^{\prime \prime}\right)}{E+E^{\prime \prime \prime}} .
\end{aligned}
$$

All the terms contained in $\mathscr{V}^{(1)}$ come from the virtual excitations from the intermediate states $A_{s}^{+}\left|\Psi_{0}\right\rangle$ and $A_{c}^{+}(E)\left|\Psi_{0}\right\rangle$ to the states $A_{c}(E)\left|\Psi_{0}\right\rangle$ via the boson-boson interaction. Those states then must decay back to the states where they are excited from. As a consequence, the term $\mathscr{V}^{(1)}$ is a term of higher order in the interaction $V$. It is at least of 
third order in $V$ and then we drop it. Gathering the results, we get a simplified formula for the optical-model potential

$$
\begin{aligned}
\mathscr{V}_{E}^{\text {opt }(c)}\left(E^{\prime}, E^{\prime \prime}\right)= & v_{0}\left(E^{\prime}, E^{\prime \prime}\right)+V_{c c}^{(1)}\left(E^{\prime}, E^{\prime \prime}\right) \\
& -i \pi \sum_{c^{\prime} \neq c} \zeta_{c c^{\prime}}^{(1)}\left(E^{\prime}, E\right) \zeta_{c^{\prime} c}^{(1)}\left(E, E^{\prime \prime}\right)-i \pi \frac{\overline{\gamma_{s c}\left(E^{\prime}\right) \gamma_{s c}\left(E^{\prime \prime}\right)^{s}}}{d} \\
& -\sum_{c^{\prime} \neq c} \int_{\varepsilon_{c^{\prime}}}^{\infty} d E^{\prime \prime \prime} \frac{V_{c c^{\prime}}^{(2)}\left(E^{\prime}, E^{\prime \prime \prime}\right) V_{c^{\prime} c}^{(2)}\left(E^{\prime \prime \prime}, E^{\prime \prime}\right)}{E+E^{\prime \prime \prime}} \\
& -\sum_{s} \frac{V_{s c}^{(2)}\left(E^{\prime}\right) V_{s c}^{(2)}\left(E^{\prime \prime}\right)}{E+E_{s}}
\end{aligned}
$$

We see that the main consequence of the introduction of correlations in the ground state is the modification of the inelastic channels form factors $\zeta_{c c}^{(1)}\left(E^{\prime}, E^{\prime \prime}\right)$ and the compound states form factors $\gamma_{s c}\left(E^{\prime}\right)$ which reduce respectively to $V_{c c^{\prime}}^{(1)}\left(E^{\prime}, E^{\prime \prime}\right)$ and $V_{s c}^{(1)}\left(E^{\prime}\right)$ in the TDA. We discuss briefly and qualitatively how these form factors are influenced by the RPA. The work of Dover and Hahne ${ }^{9}$ indicates that within the RPA model described here the resonant state partial widths are not enhanced and even slightly damped by the correlations. Since the partial width in channel $c$ of the resonant states of energy $E_{s}$ is given approximately by $\gamma_{s c}\left(E_{s}\right)$, at least for the weak coupling case, one can say that collective excitations do not enhance the resonant state form factors.

Inelastic channels form factors do not seem enhanced by the collective excitations either. In Eq. (4.13) the second term of the r.h.s. involves a "continuum Green function" evaluated at a negative value of its argument:

$$
G_{c}\left(r, r^{\prime},-E\right)=\int_{\varepsilon_{c}}^{\infty} d E^{\prime}-\frac{u_{c}}{\left(r^{\prime}, k\right) u_{c}\left(r^{\prime}, k^{\prime}\right)} \text {. }
$$

This quantity is probably very small. In fact, the RPA model of Ref. ${ }^{8}$ which is retained here provides a poor description of the collective states of the target. Since those are more or less given by

$$
\left|\chi_{E}^{c}\right\rangle=\bar{a}_{h}^{+}\left|\Psi_{0}\right\rangle
$$

all of them contain the same correlations except for a hole. To remedy to this situation, one has first to construct operators $q_{E}^{c^{+}}$and $q_{E}^{c}$ following (2.6) by taking in $\mathscr{H}$ that part of the interaction which scatters particles in bound orbitals only, i.e. by taking in Eq. (2.4) matrix elements $V_{c c^{\prime}}^{(1)}$ and $V_{c c^{\prime}}^{(2)}$ with equal $E^{\prime}$ and $E^{\prime \prime}$ variables. The operators $q_{E}^{c^{+}}, q_{E}^{c}$ create (annihilate) a particle in the continuum and a correlated hole in the ground state $\Psi_{0}$. The investigation of such a RPA model remains to be done, as far as the optical-model potential is concerned. 


\title{
5. Conclusions
}

We have studied theoretically an optical-model potential in the framework of the RPA equations extended to the continuum in order to exhibit the effect of the correlations in the ground state and hence the effect of the collective excitations on the absorption. This effect is not easy to visualize because it is mixed with two other effects: the effect of the continuum-continuum interaction and an effect proper to the RPA. Indeed, as we have shown, some boson states conjugated to the channel states do not contribute to the asymptotic behaviour of the wave function and hence induce intermediate virtual excitations. Nevertheless, it can be said that the inelastic channels and resonant states contributions to the imaginary part of the optical-model potential differ from what they are in the TDA. They reflect the importance of the correlations in the ground state. However, in practice, it seems that this does not lead to an enhancement of the imaginary part. This is partly explained by the fact that all the target states contain the same correlations except for a hole. Moreover, collective excitations introduce slowly varying terms in the optical-model potential. They are due to virtual excitations to the conjugate of the compound nucleus states and to the inelastic channel states.

We want to thank Prof. C. Mahaux for his kind interest and helpful discussions.

\author{
J. Cugnon \\ Theoretical Nuclear Physics \\ University Liège \\ Sart Tilman \\ 4000 Liège 1/Belgien
}

